\title{
Token Finding Using Mobile Agents
}

\author{
Delbert Hart ${ }^{1}$, Mihail E. Tudoreanu ${ }^{2}$, and Eileen Kraemer ${ }^{3}$ \\ 1 University of Alabama in Huntsville \\ Huntsville, AL 35899 USA \\ dhart@cs.uah.edu \\ 2 Washington University in St. Louis \\ St. Louis, MO, 63130 USA \\ renu@cs. wustl.edu \\ 3 University of Georgia Athens, GA, 30606 USA \\ eileen@cs.uga.edu
}

\begin{abstract}
One of the greatest challenges facing the software community today is the increasing complexity of software. Complexity limits understanding, making it difficult to evaluate the correctness, reliability, and performance of a system. Coupled with visualization, monitoring can provide users with insight into an application's behavior. Monitoring can also be used in conjunction with automated tools to adaptively tune performance. This paper presents a detailed look at how mobile agents that are embedded in a monitoring system can be used to find a token within a distributed system. Several strategies by which agents may accomplish this task are qualitatively compared. We then describe tests that were performed to evaluate trade-offs among the strategies considered, and discuss the results of those tests.
\end{abstract}

\section{Introduction}

Software systems are some of the most complex constructs created by man. This degree of complexity makes it difficult to evaluate their correctness and performance a priori. Monitoring provides a practical way of learning about distributed computations. To be effective, online monitoring must adapt to the changing needs of the user while minimizing the effect monitoring has on the application. In the case of distributed applications, lag, non-determinism and the lack of a global clock further complicate the monitoring task.

This paper explores the use of mobile agents to address the challenges of monitoring distributed computations. The use of mobile agents has a number of benefits: 1) Responsiveness - agents are able to react locally to conditions at the application processes. 2) Transience - the ability to deploy agents on demand helps to minimize the overall cost of monitoring. 3) Customization agents may be encoded at run-time; and thus can make use of applicationspecific information, permitting efficient solutions. 4) Mobility - the ability to migrate between processes makes agents well suited to distributed applications in which properties are not necessarily bound to one process. 
The idea of using mobile code for monitoring tasks has been proposed by many. Yet with the exception of agents within PathFinder [5], the only other general purpose monitoring system we are aware of that intends to support mobile code is the BRISK system[1], which has plans to employ a Scheme-based language for agents. Hence, actual experience with mobile code in general purpose monitoring systems is very limited. Other uses of agents for monitoring, such as in JAT 4 and WHERE[3, do not support the migration of agents between processes of the computation. Thus, these agents lack the ability react locally and to track non-local properties as easily.

General purpose mobile agent systems, such as AgentTcl[7], are not wellsuited for the tasks of monitoring because they provide high level services such as name services, authentication, and/or network references that are not necessary for monitoring and incur extra perturbation. General purpose systems are typically designed to serve as stand-alone systems instead of being embedded within another application or library. For these reasons, we have designed and implemented a simple mobile code model, specialized for the purposes of monitoring and steering activities of distributed computations.

This model is used as the basis for implementing specific mobile agent modules that are part of a larger monitoring and steering framework, the PathFinder [5] exploratory visualization system. Within the wide range of environments and tasks in a monitoring system, agents may employ several strategies to perform the monitoring task. To illustrate the richness of agent solutions this paper presents an example monitoring task, finding a token in a distributed system. We provide a novel analysis of how mobile code can be used to address this problem. We then perform experiments designed to highlight the trade-offs between two specific strategies that represent the extremes of mobility versus non-mobility, providing insight as to when mobile agents are appropriate. Section 2 gives a brief description of the PathFinder exploratory visualization system and how mobile agents are supported. The token finding example and experiments are given in Sect. 3. The results of the experiments and discussion are provided in Sect. 4 .

\section{PATHFINDER}

PathFinder manages the size and complexity of distributed systems by engaging the user as an active partner who guides both the collection of data from the application and the visual representation of the program's state and behavior. The key insight is that it is both unnecessary and inefficient to collect all possible data that an application can provide. Instead, one should collect only the data that supports the user's current interests. This selective monitoring, in conjunction with navigation tools to modify the viewer's perspective on the computation, provides a dynamic and interactive paradigm for monitoring distributed systems. The use of mobile code to realize these interactions is a natural extension of this exploratory visualization approach. 


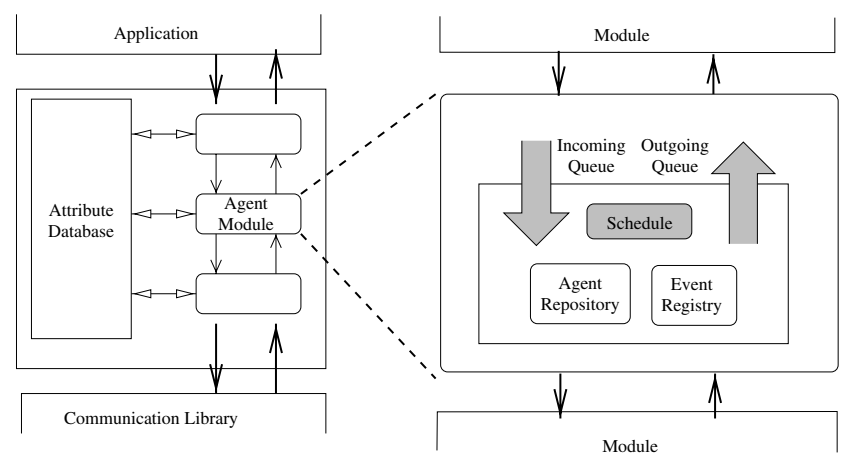

Fig. 1. An agent module can be one of many modules installed to provide monitoring and steering related services. The agent module provides the bridge between the application and the milieu.

\subsection{Agent Model}

The agent model has two kinds of entities: agents and milieux (agent servers). The distinguishing feature of this model is that it is specialized to be embedded within a monitoring library. This constrains the milieux to potentially sharing the thread of control with the application and utilizing an abstract asynchronous form of communication with other milieux. The former constraint arises from the languages being monitored. The latter is due to the fact that PathFinder is designed to be applicable to computations using any form of message passing. Thus, no assumptions about the interprocess communication is made other than its existence. Agents access information about the application via specialized agents called avatar agents and event agents, providing synchronous and asynchronous access, respectively. The milieu provides services that allow an agent to execute within it, to interact with other agents, and to move to other milieux. A milieu does not support any form of inter-milieux communication other than agent migration.

Each agent is an encoding of data and code. The code contained within an agent is stored as a set of handlers. These methods on the agent define how it reacts to events in the milieu. A distinguished handler of the agent is the Arrival handler which is automatically executed by the milieu when the agent arrives. An agent is considered to be active while one of its handlers is executing. If the agent migrates to another milieux all of its state information is included in the transfer. When an agent's handler completes execution the agent becomes dormant, waiting until one of its handlers is triggered.

The milieu (Fig. 1) interacts with the outside world via incoming and outgoing queues, which contain agents. When an agent arrives at a milieu, it is removed from the incoming queue and placed in the agent repository, where it remains while it is in the milieu. Internally, a milieu is driven by events. Events are signaled either by an agent or by the arrival of an agent via the incoming queue. Agents register to react to an event by indicating which of their han- 
dler(s) should be triggered. The milieu uses the event registry to schedule agent handlers in response to the signaled event. The handler is executed atomically, and receives the agent that signaled the event as an argument.

From within a milieu, agents are able to interact with other local (in the same milieu) agents and with the milieu itself. A milieu allows an agent to: 1) Access any other agent in the milieu through the agent repository. The fields or handlers of any agent, including itself, can be read from or written to. 2) Signal events. Further, an agent may insert or remove entries of the event registry pertaining to any agent. 3) Create a new agent. 4) Induce an agent to move to another milieu. For performance reasons the model does not address issues, such as security, that are orthogonal to using agents for monitoring and steering.

\subsection{Implementation}

One instantiation of the model uses Perl to construct the internals of the milieu (a PMilieu) and to serve as the base language for agents. Perl[8] is an objectoriented, imperative scripting language. The interface between the Perl portions of the PMilieu and the $\mathrm{C}$ coded portions consists of bindings of the incoming and outgoing queues, Perl modules that provide interfaces to resources present at the monitored process, and event agents that are generated by the agent module and inserted into the incoming queue of the milieu. SWIG 2] was used to create the extension modules that provide access to the resources of the process. Control is transferred to the PMilieu when an event occurs within the process. The application regains control after all of the agents have responded to the event, represented by an event agent.

Other implementations of agent modules for PathFinder support different styles of agents written in other languages. However, all of the agent modules are based on the same model, allowing reasoning about using mobile agents without going into specifics of the implementation and facilitating cooperation between agents in different modules.

\section{Token Finding}

\subsection{Strategies}

One use of mobile agents is to discover resources available in a wide-area network [6. The resource discovery task is similar to finding a particular property in a distributed application. Throughout this section we will refer to the property as a token, but it could be any property over the local state of a process. A token enables the process holding it to perform some action(s), and can be used as a form of coordination among processes. Finding a token in a distributed system is a common task in monitoring. Data obtained by monitoring the process that holds the token can help to provide insight into the coordinated action. Mobile agents may be used to locate and continuously track a token as it moves from one process to another. 
Each strategy begins with an initial process, called the coordinator, which initiates the token-finding process. If a process has an agent at it, then the process is said to be covered by the monitoring. Nodes are marked as clean when no token is present and no token is on the way to the process. We consider different strategies for token finding (Table11).

wait The monitoring agent remains at a node until the token arrives there.

flood The coordinator sends an agent to one node. Upon receiving an agent, the node sends the token out on all of its links. This repeats until all nodes and links have been affected.

broadcast The coordinator sends a monitoring agent directly to all other nodes. Broadcast affects only one link per application node, in contrast to flood which affects all links.

search The monitoring agent moves from one node to another until the token is found. The movement may be directed or undirected. Only one copy of the agent exists.

entrapment Agents cooperate to partition the network, and then watch for the token at the boundary nodes, and slowly "tighten the net".

wavefront Similar to entrapment, the monitoring agent starts from one locations and sends out a wave of agents looking for the token.

time to live Similar to the search strategy, but the monitoring agent is assigned a maximum number of hops before it ends.

For these strategy the properties of growth, propagation, residue, and guarantees are examined. The properties differ in how the monitoring coverage changes over time. The growth property indicates how quickly the coverage can increase. The residue property indicates how long a node remains part of the coverage and the propagation property measures how far the coverage extends. Table 2 summarizes how the strategies in Table 1 differ.

Growth Each strategy begins the token finding process at some initial set of processes and then extends coverage until the token is found. The growth property describes how the coverage grows, how monitoring spreads from one process in the computation to another without intervention by the coordinator. In strategies such as wait and broadcast, processes do not interact with each other; hence, there is no growth. The search strategy also does not grow. Instead, it moves the monitoring agent from one process to another.

The entrapment strategy adds one process at a time to the coverage area, exhibiting constant growth. Proportional growth strategies scale their expansion based on the size of the computation or the local number of links at the process. The flood strategy activates monitoring at all neighbors of a process, exhibiting proportional growth.

A trade-off exists between the rate of growth of a strategy and the resource usage of the algorithm. A high growth rate will reduce the amount of lag between the request for finding the token and the time of its location. However, a computation may not be able to tolerate the resource demands of a high growth rate. Another consideration is that strategies with growth rates above a low constant, e.g. one, are difficult to halt before they have checked the whole network. Even if 
Table 1. Example token finding strategies.

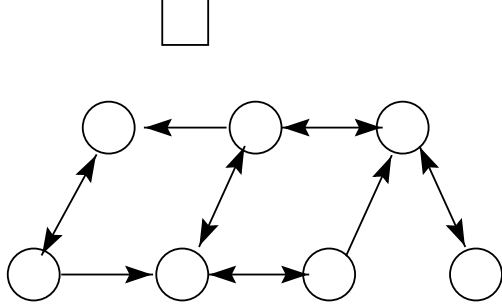

For simplicity, the examples will assume the network is fixed and FIFO. The circles represent nodes of the network and the arrows directional channels. The coordinator is shown as a square.

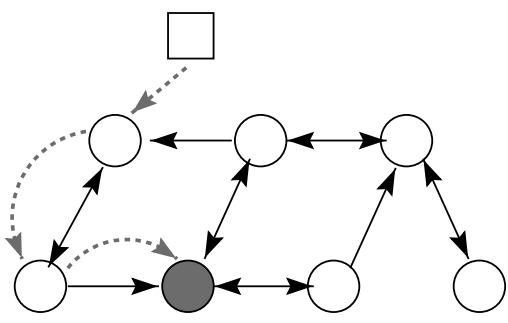

(search) Instead of waiting for the token to arrive the search strategy moves from node to node looking for the token. The search ends after a predetermined number of nodes have been visited.

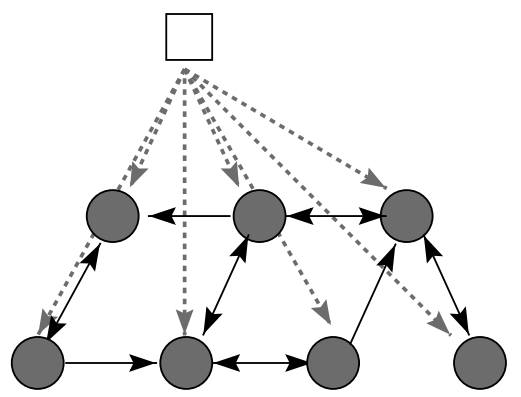

(broadcast) The coordinator directly activates monitoring in all of the nodes in the broadcast strategy. After a period of time the monitoring automatically deactivates.

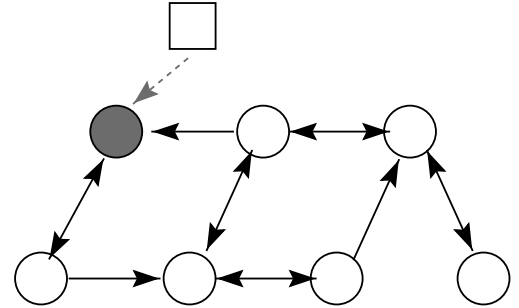

(wait) A simple strategy is to activate monitoring at a single node and wait for the token to arrive.

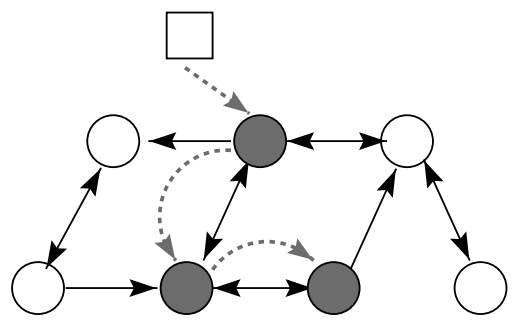

(entrapment) An entrapment strategy seeks to increasingly limit the token's possible locations until it is found. In this example the entrapment strategy has partitioned the network into two parts, watching the nodes in the middle to ensure that the token does not move into the left half while it is searching the right half.

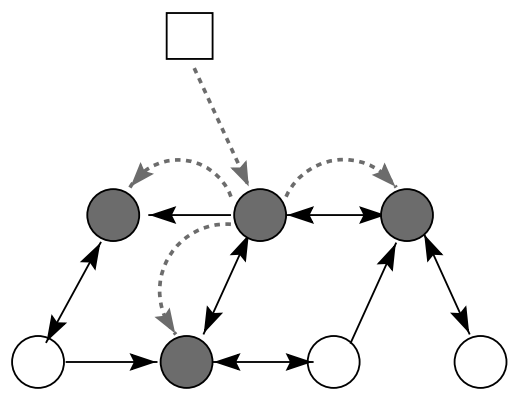

(flood) The flood strategy begins at one node and spreads on all links until the entire computation is looking for the token. 
Table 2. Characteristics of different token finding strategies.

\begin{tabular}{|l||c|c|c|c|c||c|c||c|}
\hline \multicolumn{1}{|c|}{} & \multicolumn{3}{c||}{ Growth } & \multicolumn{2}{c||}{ Residue } & Propagation & Guaranteed \\
& None/One & Constant & Proport. & expire & explicit & finite & infinite & to find \\
\hline \hline wait & $\mathrm{x}$ & & & & $\mathrm{x}$ & $\mathrm{n} / \mathrm{a}$ & $\mathrm{n} / \mathrm{a}$ & $\mathrm{no}$ \\
\hline search & $\mathrm{x}$ & & & $\mathrm{n} / \mathrm{a}$ & $\mathrm{n} / \mathrm{a}$ & $\mathrm{x}$ & & $\mathrm{no}$ \\
\hline entrapment & & $\mathrm{x}$ & & & $\mathrm{x}$ & & $\mathrm{x}$ & $\mathrm{yes}$ \\
\hline broadcast & $\mathrm{x}$ & & & $\mathrm{x}$ & & $\mathrm{n} / \mathrm{a}$ & $\mathrm{n} / \mathrm{a}$ & yes \\
\hline flood & & & $\mathrm{x}$ & & $\mathrm{x}$ & & $\mathrm{x}$ & yes \\
\hline
\end{tabular}

the token is found almost immediately, there is no way to quickly communicate this information to halt the growth of coverage.

Residue Residue refers to how long artifacts from the strategy remain at a process. After initially determining that the token is not present at a process, most strategies will continue to watch the process for a time to ensure that the token does not arrive from one of the channels. Some strategies deactivate monitoring if it has been determined that the token can not reach the process undetected, such as in the case in which all channels arriving at the process have been flushed and the sources are being monitored.

Flushing lets a process know that a token is not in an incoming channel. Flushing depends upon the communication characteristics of the network. The broadcast strategy does an implicit flush of the channels. It knows that monitoring will be active at all processes by a certain time. Then it simply waits until all messages sent by that time would have been delivered. It assumes a maximum network delay exists, which is reasonable in many environments. Explicitly flushing a channel consists of sending a marker or message to indicate that the token is no longer in the channel. Once markers have been received on all channels, the process may safely deactivate its monitoring. This technique assumes that the channels are at least FIFO.

Explicit flushing has less lag, but a higher message overhead and typically involves a more complicated scheme to both clean-up and to keep message traffic at a reasonable level. Another trade-off to consider is between having long lasting residuals and the growth and propagation of the coverage. The slower growing and further propagating strategies often will have long lasting residuals. On the other hand a fast growing strategy may have a short residual (or none at all). One relevant factor in this decision is how much overhead the residual imposes on the process. That is, how expensive it is to watch the incoming messages and/or periodically check the computation's state for the token. Also of concern is how sensitive the computation is to message traffic.

Propagation \& Guarantees Propagation is related to the rate of growth of a strategy and is related to how far the coverage extends from its point of origin. Some algorithms stop propagating after reaching some finite measure, such as a time limit or a hop-count. Other algorithms will continue until the token is found, no matter how long that takes. Performance or time bounds may 
exist on finding the token. For example, token finding may cease if the token is not found by the time the computation enters the next phase. The most common guarantee of a strategy is that if it is run to completion, then the token will be found. The entrapment and flood strategies guarantee that the token will be found, while strategies like wait and search do not make this guarantee. Other examples of guarantees made by a strategy might describe how resources will be utilized by it.

\subsection{Train Simulation}

To demonstrate the trade-offs between the different token finding strategies, a railroad simulation is used. The computation consists of a "smart" railroad that simulates algorithms that ensure the safe movement of trains. Components of the simulation are tracks, lights and switches. Each component represents a computing device capable of communicating with other components and making decisions that regulate the movement of trains. Each component is simulated by a separate process. Processes communicate only through message passing. A train moves from one component to the next via a message exchange between the two components. To allow user interaction with the simulation as it runs, each component performs a one second sleep after it processes incoming messages.

\subsection{Experiments}

The token in these experiments is the locomotive of the train. The experiments considered four different variables: agent strategy, distribution, topology, and number of components.

The test-bed consisted of a primary cluster of 22 Linux workstations and a remote cluster of 4 Linux workstations about 600 kilometers away. The machines were of various configurations and speeds. Two different configurations of the test-bed were used. The first configuration used all 22 machines in the primary cluster. The second configuration used 18 machines in the primary cluster and all 4 machines in the remote cluster. The coordinator for the search was on the remote cluster in the second configuration.

Two topologies of the railroad components were used. The first topology was of a general graph that was randomly generated. The graph was constructed to be connected. That is, any component is reachable from any component. Some track components were changed to switch components to create circuits and redundant paths that are typical of real world railroads. The second topology consisted of only straight track components. The tracks were configured so they formed a ring. The total number of components in the simulation was varied from 22 to 176 .

Two different types of agents were used, based on the broadcast and search strategies. The residue in both cases was limited to the time required for the agent to check if the train was present at the component. Upon arrival at a component, an agent checks whether the locomotive is present and immediately dies or leaves that component depending on the strategy. When the locomotive 
was found the coordinator was signaled. For the broadcast strategy, the coordinating process sent the same agent to all railroad components. In the search strategy the coordinator sent an agent to a randomly chosen component. The search agent was provided with a list of all components and visited each one in order until the train was found. A timeout value was chosen after which point the train was considered to be not found.

\section{Results and Discussion}
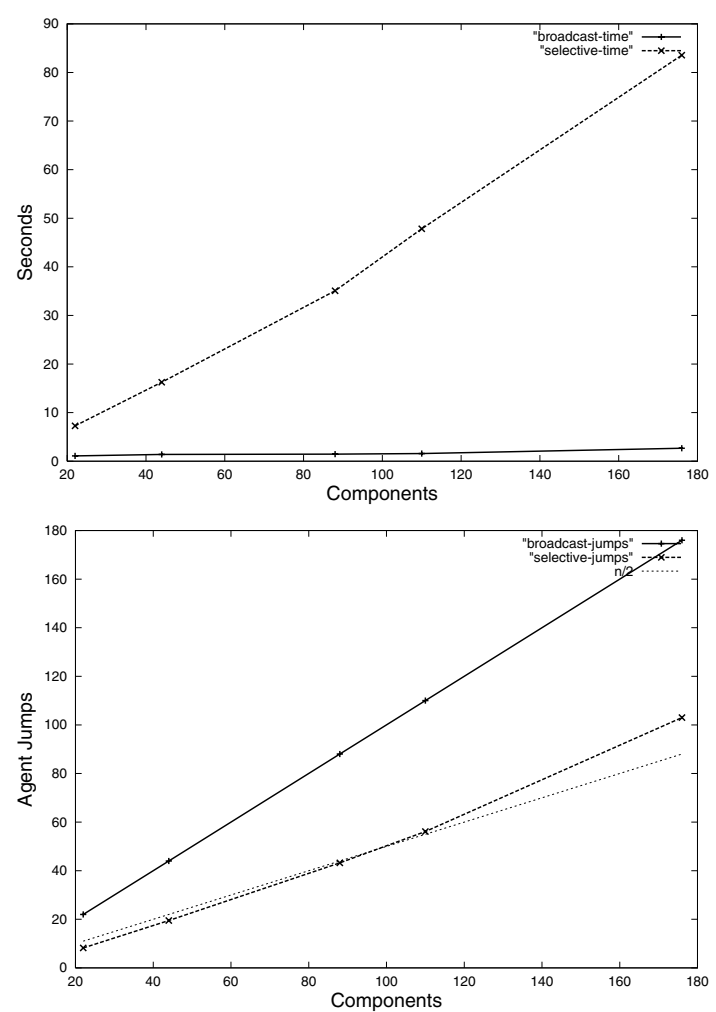

Fig. 2. (Top) The amount of time to find the token is plotted for various number of processes. The amount of time the search strategy took was linear in the number of components compared to the broadcast strategy which was relatively constant. (Bottom) For both strategies, the number of agent jumps is linear relative to the number of components. The search strategy uses approximately half the number of jumps of the broadcast strategy.

Approximately 150 tests were executed. No significant difference was found based on the topology or test-bed configuration. The one second delay in the 
application effectively hid any difference in the latency of agent transmittals between the two clusters. The train was not found by the agents during five tests - one a broadcast strategy and four search strategies. This happened because the train either was in transit when the agents were sent out or because the train moved to a component that had already been searched. If a longer residual had been provided for the broadcast strategy it would not have missed the token in that one instance.

The difference between the two strategies in terms of latency and number of agent transmittals is shown in Fig. 2, As expected, the latency for the broadcast strategy was close to constant and was linear in the number of components for the search strategy. The effect of the one second pause in the application processes can be easily noticed in the amplitude of the growth for the search strategy. Also, as expected, the number of agent transmittals for the search strategy was approximately half of what was needed for the broadcast strategy. Since the amount of overhead invoked by both types of agents was approximately the same, the perturbation of the search strategy is about half that of the broadcast strategy. The number of agent jumps by the search strategy was actually above half the number of components. This was due to the instances in which a token moved to a component that the agent had already checked.

Using different agent strategies allows for a simple way to customize what costs will be incurred during a monitoring session. The specific trade-off that we examined here was a fast, expensive, and bursty pattern of resource usage, versus a slower, cheaper, and more regular pattern. The choice that is appropriate will depend on the needs of both the user and the application. Utilizing mobile agents allows for this choice to be made easily at run-time. Further experiments are planned to quantify the cost of using agents in the monitoring activity and to further verify our qualitative analysis of the agent trade-offs.

\section{References}

[1] Bakic, A.M., Mutka, M.M., Rover, D.T.: BRISK: A Portable and Flexible Distributed Instrumentation System. Software: Practice and Experience. 12 (2000) 1353-1373

[2] Beazley, D.M.: SWIG: An easy to use tool for integrating scripting languages with $\mathrm{C}$ and $\mathrm{C}++$. Proceedings of the 4th USENIX Tcl/Tk workshop. (1996) 129-139

[3] Brooks, C., Tierney, B., Johnston, W.: JAVA Agents for Distributed System Management. Technical report, Lawrence Berkeley National Laboratory. (1998)

[4] Frost, H.R.: Documentation for the Java(tm) Agent Template. Stanford University. (1996)

[5] Hart, D., Kraemer, E., Roman, G.C.: Consistency Considerations in the Interactive Steering of Computations. International Journal of Parallel and Distributed Systems and Networks. 3 (1999) 171-179

[6] Jun, K., Boloni, L., Palacz, K., Marinescu, D.C.: Agent-Based Resource Discovery. Proceedings of 9th Heterogeneous Computing Workshop. (2000)

[7] Rus, D., Gray, R., Kotz, D.: Transportable Information Agents. Readings in Agents. (1998) 283-291

[8] Schwartz, R., Wall, L.: Programming Perl. (1994) 